\title{
Invarianza factorial por sexo del cuestionario para las conductas disruptivas y escala de evaluación de la competencia docente en educación física en estudiantes de secundaria
}

\author{
Marina MARTÍNEZ-MOLINA ${ }^{1}$, Antonio GRANERO-GALLEGOS ${ }^{1}$, \\ Antonio BAENA-EXTREMERA ${ }^{2}$ y Raúl BAÑOS ${ }^{3}$ \\ ${ }^{1}$ Universidad de Almería \\ ${ }^{2}$ Universidad de Granada \\ ${ }^{3}$ Universidad Autónoma de Baja California, México
}

(Recibido el 18 de Febrero de 2018, Aceptado el 24 de Febrero de 2020)

RESUMEN: Este trabajo tuvo como objetivos: analizar la invarianza factorial por sexo del Cuestionario de Conductas Disruptivas en el aula de Educación Física y de la Escala de Evaluación de la Competencia Docente en Educación Física, así como analizar las diferencias según la variable sexo en las subescalas de dichos instrumentos. El diseño de investigación fue observacional y descriptivo de carácter transversal y la selección de la muestra fue de tipo no probabilístico y por conveniencia. Participaron 410 estudiantes (202 chicos; 208 chicas; $M_{\text {edad }}=15.14 ; D T=.96$ ) de secundaria. Se utilizaron las siguientes escalas: Evaluación de la Competencia Docente en Educación Física y Cuestionario para las Conductas Disruptivas en Educación Física. Los modelos para el análisis de la invarianza fueron evaluados siguiendo la propuesta metodológica avanzada de testar cuatro modelos sucesivamente más constreñidos. El análisis de las diferencias por sexo fue realizado con Modelos Lineales de Regresión procedimiento MIXED. Los resultados revelaron a los dos instrumentos como válidos para medir los comportamientos negativos de los estudiantes y la competencia docente, independientemente del sexo del alumnado. Los chicos obtuvieron puntuaciones significativamente más altas en conductas disruptivas que las chicas, mientras que ellas percibieron más competente al profesorado.

Palabras clave: mal comportamiento; alumnado; adolescencia; profesorado.

Factorial invariance by sex of the Physical Education Classroom Instrument and Evaluation of Teaching Competencies Scale adapted to Physical Education with secondary students

ABSTRACT: This work had the following aims: to analyze the factorial invariance by sex of the Physical Education Classroom Instrument and the Evaluation of Teaching Competencies Scale in Physical Education, as well as to analyze the differences according to the variable sex in the variables of these instruments. The research design was observational and descriptive of a transversal nature and the sample selection was non-probabilistic and for convenience. 410 students from Secondary Education 
(202 boys; 208 girls; $M_{\text {age }}=15.14 ; D T=.96$ ) participated. The following scales were used: Physical Education Classroom Instrument and Evaluation of Teaching Competencies Scale in Physical Education. The models for the analysis of the invariance were tested following the advanced methodological proposal to test four successively more constrained models. The analysis of the differences by sex was performed with Linear Models of Mixed Regression. The results showed the two instruments as valid to measure the negative behaviors of the students and the teaching competence, regardless of the sex of the students. The boys obtained significantly higher scores in disruptive behaviors than the girls, while they perceived the teachers as more competent.

Key words: Misbehaviour; Student; Adolescence; Teacher.

Correspondencia: E-mail: agranero@ual.es

\section{Introducción}

El mal comportamiento en las aulas por parte del alumnado de secundaria constituye un grave problema educativo en la actualidad (Medina y Reverte, 2019), lo que puede tener consecuencias en la motivación, satisfacción con la escuela y el fracaso escolar por parte de los adolescentes o hasta en la relación con el profesorado (Baena-Extremera, Granero-Gallegos, Pérez-Quero, Bracho-Amador, y Sánchez-Fuentes, 2013; Baños, Ortiz-Camacho, Baena-Extremera, y Tristán-Rivera, 2017; Granero-Gallegos, Gómez-López, Abraldes, y Rodríguez-Suárez 2011; Granero-Gallegos, Gómez-López, Baena-Extremera, Martínez-Molina, 2019). Como ponen de manifiesto algunos estudios (Baena-Extremera, Granero-Gallegos, y Martínez-Molina, 2015; Colinchón, 2020; Goyette, Dore, y Dion, 2000; Kulinna, Cothran, y Regualos, 2006), los adolescentes a menudo muestran algunos comportamientos problemáticos en las aulas, como ociosidad, falta de respeto, irresponsabilidad, desobediencia de las normas, hablar fuera de turno, y/o eluden o faltan a clase, lo que genera un impacto negativo en el entorno de aprendizaje.

Estas conductas disruptivas también están presentes en las aulas de Educación Física (EF) (Baena-Extremera et al., 2015; Weiss, Smith, y Stuntz, 2008), produciendo situaciones conflictivas entre estudiantes y hasta con el propio docente, por lo que es recomendable solucionar la problemática de una forma rápida y eficaz (Müller, Hofmann, Begert, y Cillessen, 2018). Ante este tipo de situaciones, las competencias del profesorado adquieren un papel importante (Baños et al., 2017), pues el diseño, organización y control de las sesiones de clase pueden relacionarse con los comportamientos disruptivos.

Los estudios sobre las conductas inadecuadas en EF han demostrado que el comportamiento negativo de los estudiantes no solo afecta a la calidad de la enseñanza, sino que también interfiere con el aprendizaje entre iguales (Cothran, Kulinna, y Garrahy 2009; Kulinna, Cothran, y Regualos, 2006). Además, en la etapa de educación secundaria son más comunes los comportamientos disruptivos que en educación primaria (ver e.g., Cothran y Kulinna, 2007; Kulinna et al., 2006). Dado que la adolescencia se caracteriza por ser una etapa de rebeldía, inconformismo, lucha contra lo estipulado, irresponsabilidad y bajo autocontrol personal, en esta etapa se puede producir una desvinculación de la escuela, disminuyendo la disposición a cumplir con las reglas y las expectativas del comportamiento (Fredricks, Blumenfield, y Paris, 2004). Estos aspectos ponen de manifiesto, más si cabe, la importancia de estudiar los malos comportamientos en las aulas.

(C) Psy, Soc, \& Educ, 2020, Vol. 12(2) 
Una de las variables que se utilizan para explicar y estudiar las conductas disruptivas es el sexo de los estudiantes. Diversos estudios han encontrado mayores niveles de conductas inapropiadas entre los chicos que entre las chicas (Beaman, Wheldall, y Kemp, 2006; Cothran y Kulinna, 2007; Kulina et al., 2006). Los varones en estas edades adolescentes suelen ser más bulliciosos, disruptivos y perturbadores (Glock y Kleen, 2017), mientras que las chicas suelen ser más proactivas y menos problemáticas (Driessen, 2011). No obstante, otros autores (i.e., Baños, Ortiz-Camacho, Baena-Extremera, y Zamarripa, 2018) sí encontraron que las chicas afirmaban tener más comportamientos agresivos en las sesiones de EF.

Uno de los instrumentos utilizados en España para el estudio del mal comportamiento en las aulas es el Cuestionario para las Conductas Disruptivas en Educación Física (CCDEF) de Granero-Gallegos y Baena-Extremera (2016), adaptación al contexto español de educación secundaria de la escala original, Physical Education Classroom Instrument (PECI) de Krech, Kulinna, y Cothran (2010). No obstante, a pesar de la solidez de las propiedades psicométricas demostradas en la validación de esta referenciada versión española y su uso en estudios actuales (Granero-Gallegos et al, 2019a; Granero-Gallegos, Ruiz-Montero, Baena-Extremera, y Martínez-Molina, 2019; Granero-Gallegos, Baños, Baena-Extremera, y Martínez-Molina, 2020), es necesario realizar un análisis de invarianza factorial entre diferentes muestras (i.e., sexo), para el estudio de las diferencias según el sexo de los estudiantes. Esta escala permite medir en el alumnado las conductas de: agresividad, irresponsabilidad y bajo compromiso, desobediencia de las normas, perturbación del ambiente de clase, y bajo autocontrol personal.

Entre las atribuciones de los estudiantes a los comportamientos inapropiados en el aula de EF destaca el aburrimiento que vivencian en sus clases, encontrándolas monótonas, además de manifestar un cierto descontento con el docente; no obstante, en ocasiones hay que destacar que se trata de alumnos con conductas disruptivas habituales (Cothran y Kulinna, 2007). Este aspecto hace más importante aún el papel del profesor y su competencia a la hora de enseñar, dado que, como indican Cothran et al. (2009), una ineficaz gestión del aula provoca descontrol e interrupciones producidas por unos pocos adolescentes, que afectan tanto a la ansiedad y al estrés de sus compañeros como a la del profesorado. Además, en relación con el profesorado, algunos estudios han relacionado las conductas disruptivas del alumnado de EF con las competencias docentes (i.e., Baños et al., 2019; Granero-Gallegos et al., 2019a; Granero-Gallegos et al., 2019b). Se destaca la importancia de la competencia del profesor, percibida por los estudiantes, para la disminución de los malos comportamientos. Por tanto, la labor del docente en EF adquiere un papel importante en el desarrollo de unas buenas conductas en el aula y en función de las habilidades docentes podrán aumentar o disminuir los comportamientos negativos en el aula (Granero-Gallegos et al., 2019b; Rasmussen, Scrabis-Fletcher, y Silverman, 2014).

Para estudiar la competencia docente percibida por parte del alumnado de EF, uno de los instrumentos utilizados en España es la Escala de Evaluación de la Competencia Docente en Educación Física (ETCS-EF) de Baena-Extremera et al. (2015), adaptada del instrumento original de Catano y Harvey (2011), Evaluation of Teaching Competencies Scale. La validación española de este instrumento ha demostrado unas excelentes propiedades psicométricas para medir los requisitos que el profesor debe cumplir para ser competente: buena comunicación, conciencia de trabajo, creatividad, feed-back, consideración individual, profesionalidad, resolución de problemas y conciencia social. Por ello, se hace necesario llevar a cabo un análisis de invarianza 
factorial entre diferentes muestras (i.e., sexo), para el estudio de las diferencias según el sexo de los estudiantes.

A partir de lo expuesto en los párrafos anteriores, el presente trabajo se plantea los siguientes objetivos: i) Analizar la invarianza factorial por sexo del Cuestionario de Conductas Disruptivas en el aula de Educación Física y de la Escala de Evaluación de la Competencia Docente en Educación Física; ii) Analizar las diferencias según la variable sexo en alumnado de educación secundaria, en las conductas disruptivas en el aula de EF y en la evaluación de la competencia docente en $\mathrm{EF}$.

\section{Método}

\section{Participantes}

El diseño de esta investigación fue observacional y descriptivo de carácter transversal y se realizó una selección de la muestra de tipo no probabilístico y por conveniencia, según el alumnado al que se pudo acceder. Participaron un total de 410 estudiantes ( 202 hombres $=49.3 \% ; 208$ mujeres $=50.7 \%$ ) de Educación Secundaria Obligatoria (ESO) de centros públicos de la Región de Murcia. Estos institutos están localizados en áreas de nivel socioeconómico medio y ninguno está incluido en programas relacionados con Educación Compensatoria con recursos específicos, materiales y humanos para garantizar el acceso, permanencia y promoción en el sistema educativo de estudiantes socialmente desfavorecidos. El rango de edad estuvo comprendido entre 13 y 16 años $(M=15.14 ; D T=.96)$, siendo la edad media de los chicos $15.11(D T=.98)$ y la de las chicas $15.17(D T=.94)$. La distribución por cursos fue de 136 estudiantes en $2^{\circ}$ de $\operatorname{ESO}(33.2 \%)$, 131 en $3^{\circ}$ de $\operatorname{ESO}(32 \%)$ y 143 en $4^{\circ}$ de $\operatorname{ESO}(34.8 \%)$.

\section{Instrumentos}

Conductas disruptivas en Educación Física. Se utilizó el Cuestionario para las Conductas Disruptivas en Educación Física (CCDEF) de Granero-Gallegos y Baena-Extremera (2016), versión española del original, Physical Education Classroom Instrument (PECI) de Krech et al. (2010). Esta versión consta de 17 ítems que miden las conductas disruptivas del alumnado en la clase de EF en cinco subescalas: agresividad (AGR) (2 ítems; e.g., "Amenazo a los demás compañeros/as de clase"), irresponsabilidad y bajo compromiso (IRR) (4 ítems; e.g., "Soy perezoso/a en clase"), desobediencia de las normas (DES) (4 ítems; "No sigo las instrucciones"), perturbador del ambiente de clase (PAC) (4 ítems; e.g., "Abandono el grupo durante una actividad"), y bajo autocontrol personal (BAP) (3 ítems; e.g., "Me burlo de otros/as compañeros/as de clase"). Para las respuestas se utilizó una escala Likert de cinco puntos que oscilaba entre 1 (nunca) y 5 (siempre). Los índices de consistencia interna fueron: agresividad, alfa de Cronbach $(\alpha)=.56$, fiabilidad compuesta $=.57$, Varianza Media Extraída (AVE, por sus siglas en inglés $)=.41$; IRR, $\alpha=.71$, fiabilidad compuesta $=.74, \mathrm{AVE}=.54$; DES, $\alpha=.75$, fiabilidad compues$\mathrm{ta}=.73, \mathrm{AVE}=.52 ; \mathrm{PAC}, \alpha=.79$, fiabilidad compuesta $=.82, \mathrm{AVE}=.58$; $\mathrm{BAP}, \alpha=.82$, fiabilidad compuesta $=.82, \mathrm{AVE}=.60$. Dados los bajos índices de consistencia interna de la subescala AGR, se desestimó este factor y se calculó el ajuste con el modelo de cuatro factores y 15 ítems (Grane- 
ro-Gallegos y Baena-Extremera, 2016) para ser utilizado en los análisis realizados. Los índices de bondad de ajuste de la escala mediante análisis factorial confirmatorio (AFC) de este modelo de cuatro factores fueron: chi-cuadrado $\left(\chi^{2}\right) /$ grados de libertad $(\mathrm{gl})=2.17$; Índice de Ajuste Incremental (IFI, por sus siglas en inglés) $=.96$; Índice de Tucker-Lewis (TLI, por sus siglas en inglés $=.95$; Índice de Ajuste Comparativo (CFI, por sus siglas en inglés)=.96; Error Cuadrático Medio de Aproximación (RMSEA, por sus siglas en inglés)=.05; Media Cuadrática del Error Tipificada (SRMR, por sus siglas en inglés) $=.04$.

Competencia docente. Se utilizó la versión española de la Escala de Evaluación de la Competencia Docente en Educación Física (ETCS-EF) de Baena-Extremera et al. (2015), adaptada del original, Evaluation of Teaching Competencies Scale (ETCS) de Catano y Harvey (2011). Consta de ocho ítems para medir la percepción de los estudiantes de la eficacia del docente. Para las respuestas se utilizó una escala Likert de siete puntos que oscila entre bajo (1, 2; e.g., "El profesor/a de EF tiene un tono monótono de voz, cuando explica depende de las notas escritas y tiene dificultades para responder preguntas de los alumnos/as"), medio $(3,4,5$; e.g., "El profesor/a de EF no imparte todos los contenidos que se indican en el programa de la asignatura y a veces parecen un poco improvisados") y alto (6, 7; e.g., "El profesor/a de EF proporciona un detallado y completo programa del curso, prepara e imparte muy bien todos los contenidos"). Los índices de consistencia interna fueron: $\alpha=.87$, fiabilidad compuesta $=.83, \mathrm{AVE}=.56$. Los índices de bondad de ajuste de la escala de competencia docente (CD) mediante AFC fueron: $\chi^{2} / g l=2.41$; $\mathrm{IFI}=.97 ; \mathrm{TLI}=.96 ; \mathrm{CFI}=.97 ; \mathrm{RMSEA}=.06 ; \mathrm{SRMR}=.03$.

\section{Procedimiento}

Se obtuvo permiso para desarrollar el trabajo por parte de los órganos competentes, tanto de los centros educativos de secundaria como universitarios. Padres/madres y adolescentes fueron informados acerca del protocolo y objeto del estudio. La firma del consentimiento informado por parte de ambos fue requisito indispensable para la participación en la instigación. Los instrumentos para medir las diferentes variables se administraron en el aula por los propios investigadores y sin la presencia del docente. Todos los participantes fueron informados del objetivo de estudio, voluntariedad y confidencialidad de las respuestas y manejo de datos, así como de sus derechos como participantes en el mismo, en base a la Declaración de Helsinki (2008). Esta investigación cuenta con la aprobación del Comité de Ética de la Universidad de Murcia (REF45-20/01/2016).

\section{Análisis de datos}

Los modelos para el análisis de la invarianza fueron realizados con AMOS 24. El resto de análisis fueron realizados con SPSS 24. La invarianza según el sexo (invarianza multigrupo o invarianza factorial) del CCDEF y del ETCS-EF fue testada siguiendo la propuesta metodológica avanzada por Milfont y Fisher (2010) de testar cuatro modelos sucesivamente más constreñidos; por ello, se han tenido en cuenta cuatro modelos anidados progresivamente más restrictivos: (1) Modelo 1, invarianza configural (M1, sin restricción), a partir del cual se comparan el resto de modelos; (2) Modelo 2, invarianza métrica (M2, restricción en cargas factoriales); Modelo 3, invarianza fuerte (M3, restricción en cargas factoriales e interceptos); Modelo 4, invarianza estricta 
(M4, restricción en cargas factoriales, interceptos y varianzas de error). Siguiendo a Chen (2007), estos modelos anidados fueron comparados teniendo en cuenta el cambio $(\Delta)$ en los índices de bondad de ajuste (i.e., incremento en el RMSEA de al menos .015 o decrecimiento en CFI y TLI de al menos .010, indican falta de invarianza) (Chen, 2007)intercepts, and residual variances. Standardized root mean square residual (SRMR.

Es importante señalar que los datos de este trabajo se recogieron en centros educativos por lo que el alumnado puede estar anidado en función del centro y curso y/o clase, es decir, violando el principio de independencia de las observaciones, por lo que se consideró conveniente utilizar Modelos Lineales de Regresión Multinivel o Mixta (MLM) (Gelman y Hill, 2007), teniendo en cuenta variables de características individuales de los participantes (nivel 1) y variables de contexto (nivel 2) (Hox, 1998). Las variables dependientes fueron las diferentes subescalas del CCDEF y ETCS-EF, y se consideró como efecto aleatorio la agrupación o nivel de centro educativo. Los análisis de regresión fueron realizados con el procedimiento MIXED de SPSS 24.0, con el método de estimación de Máxima Verosimilitud Restringida. Se utilizó el Logaritmo de la Verosimilitud -2 (-2LL) (Pardo, Ruiz, y San-Martín, 2007) para estimar los efectos de la variable centro educativo en cada modelo estimado. Se testaron diferentes modelos de regresión atendiendo a las diferentes combinaciones de los niveles de centro educativo con cada una de las variables dependientes, incluido un modelo nulo. La variable "centro educativo" resultó estadísticamente significativa $(p<.05)$ en todos los casos, por lo que se estimó que la variable de contexto "centro educativo" tiene efecto en cada modelo. Además, se calculó del Coeficiente de Correlación Intraclase (CCI) en cada una de las variables que se pretenden comparar. Los resultados mostraron que la varianza explicada era superior al $7.54 \%$ en todos los casos, lo que permite atribuir que un porcentaje de las diferencias entre las variables dependientes puede ser atribuido al centro educativo. Atendiendo a lo expuesto, las diferencias según el sexo en relación a las diferentes subescalas se calcularon mediante modelos de regresión multinivel y, en este caso, la variable independiente (factores del modelo mixto) fue sexo del alumnado.

\section{Resultados}

\section{Análisis descriptivo y de correlación}

En la Tabla 1 se comprueba que los valores de la competencia docente fueron moderadamente altos, mientras que los valores medios de las conductas disruptivas fueron moderadamente bajos, oscilando entre la irresponsabilidad y bajo compromiso y la perturbación del ambiente de clase, que presentó la media más baja.

En las correlaciones se comprueba que la competencia docente solo correlaciona de manera estadísticamente significativa, y de manera negativa, con la irresponsabilidad y bajo compromiso. Por otro lado, las conductas disruptivas presentaron correlaciones altas, positivas y estadísticamente significativas entre las propias subescalas del CCDEF, destacando los altos valores de correlación (ver Tabla 1). 
Tabla 1. Descriptivos y correlaciones de las subescalas ECTS-EF y CCDEF.

\begin{tabular}{lccccccc} 
Subescalas & $\boldsymbol{M}$ & $\boldsymbol{D T}$ & CD & IRR & DES & PAC & BAP \\
\hline CD & 5.26 & 1.07 & - & $-.13^{* *}$ & -.10 & -.08 & -.04 \\
IRR & 2.03 & .87 & & - & $.67^{* *}$ & $.59^{* *}$ & $.58^{* *}$ \\
DES & 1.70 & .82 & & & - & $.62^{* *}$ & $.62^{* *}$ \\
PAC & 1.40 & .75 & & & & - & $.77^{* *}$ \\
BAP & 1.44 & .82 & & & & & - \\
\hline
\end{tabular}

Nota. ${ }^{*} p<.05 ;{ }^{* *} p<.01 ; M=$ media; $D T=$ desviación típica; $\mathrm{CD}=$ Competencia docente; IRR = Irresponsabilidad y bajo compromiso; DES $=$ Desobediencia de las normas; $\mathrm{PAC}=$ Perturbador del ambiente de clase; $\mathrm{BAP}=\mathrm{Bajo}$ autocontrol personal.

\section{Análisis de invarianza factorial por sexo}

Se llevó a cabo la invarianza por sexo (i.e., 202 chicos, 208 chicas) del CCDEF y del ETCS-EF basado en modelos de CFA. Los resultados de las pruebas de invarianza se muestran en la Tabla 2 para el CCDEF y en la Tabla 3 para el ETCS-EF. En ambas escalas, los diferentes modelos (invarianza configural, invarianza métrica, invarianza fuerte e invarianza estricta) proporcionan un buen nivel de bondad de ajuste $(\mathrm{CFI}>.90$, TLI $>.90$, and RMSEA $<.06)$ y no exceden los puntos de corte recomendados en la comparación de modelos, cada vez más restrictivos, para $\operatorname{RMSEA}(\Delta>.015), \mathrm{CFI}(\Delta>.01)$, and TLI $(\Delta>.01)$.

Tabla 2. Análisis de invarianza por sexo para el CCDEF

\begin{tabular}{lccccccccc}
\hline Modelo & $\chi^{2}$ & $d f$ & RMSEA [90\% IC] & CFI & TLI & $\begin{array}{c}\text { Comparación } \\
\text { de modelos }\end{array}$ & $\Delta$ RMSEA & $\Delta$ CFI & $\Delta$ TLI \\
\hline 1.- M1 & $342.579 *$ & 162 & $.052[.045-.060]$ & .931 & .910 & - & & & \\
2.- M2 & $379.683 *$ & 173 & $.054[.047-.061]$ & .921 & .905 & 2 versus 1 & .002 & -.010 & -.005 \\
3.- M3 & $422.867 *$ & 188 & $.055[.048-.062]$ & .923 & .901 & 3 versus 2 & .001 & .002 & -.004 \\
4.- M4 & $342.579 *$ & 162 & $.052[.045-.060]$ & .931 & .910 & 4 versus 3 & -.003 & .008 & .009 \\
\hline
\end{tabular}

$\chi^{2}=$ Chi cuadrado; $g l=$ grados de libertad; RMSEA = Error Cuadrático Medio de Aproximación; $90 \% \mathrm{IC}=90 \%$ intervalo de confianza del RMSEA; CFI = Índice de Ajuste comparativo; TLI = Índice de Tucker-Lewis; * $p<.01$; M1 = Modelo 1, sin restricciones; M2 = Modelo 2, restricción en cargas factoriales; M3 = Modelo 3, restricción en cargas factoriales e interceptos; M4 = Modelo 4, restricción en cargas factoriales, interceptos y varianzas del error. 
Tabla 3. Análisis de invarianza por sexo para el ETCS-EF

\begin{tabular}{lccccccccc}
\hline Modelo & $\chi^{2}$ & $d f$ & RMSEA [90\% IC] & CFI & TLI & $\begin{array}{c}\text { Comparación } \\
\text { de modelos }\end{array}$ & $\Delta$ RMSEA & $\Delta$ CFI & $\Delta$ TLI \\
\hline 1.- M1 & $73.144^{*}$ & 40 & $.045[.028-.061]$ & .962 & .947 & - & & & \\
2.- M2 & $78.403^{*}$ & 47 & $.040[.024-.056]$ & .964 & .957 & 2 versus 1 & -.005 & .002 & .010 \\
3.- M3 & $87.861^{*}$ & 48 & $.045[.030-.060]$ & .955 & .947 & 3 versus 2 & .005 & -.009 & -.010 \\
4.- M4 & $112.900^{*}$ & 56 & $.050[.036-.063]$ & .945 & .939 & 4 versus 3 & .005 & -.010 & -.008 \\
\hline
\end{tabular}

$\chi^{2}=$ Chi cuadrado; $g l=$ grados de libertad; RMSEA $=$ Error Cuadrático Medio de Aproximación; 90\% IC $=90 \%$ intervalo de confianza del RMSEA; CFI = Índice de Ajuste comparativo; TLI = Índice de Tucker-Lewis; * $p<.01$; M1 = Modelo 1, sin restricciones; M2 = Modelo 2, restricción en cargas factoriales; M3 = Modelo 3, restricción en cargas factoriales e interceptos; M4 = Modelo 4, restricción en cargas factoriales, interceptos y varianzas del error.

\section{Diferencias según la variable sexo}

Para comprobar las diferencias en las subescalas de la competencia docente y de las conductas disruptivas según la variable sexo, se ha calculado el $p$-valor asociado a las pruebas estadísticas de comparación de medias marginales corregidas por múltiples comparaciones mediante SIDAK en MLM (ver Tabla 4). Los resultados indican que existen diferencias estadísticamente significativas, tanto en la percepción de la competencia docente, con valores más altos en las chicas, como en las cuatro subescalas del CCDEF, aunque en este caso, los chicos presentaron valores medios más altos en todos los factores de mal comportamiento.

Tabla 4. Diferencias según sexo en función de las subescalas ETCS-EF y CCDEF según modelo lineales mixtos

\begin{tabular}{|c|c|c|c|c|c|c|c|c|}
\hline & \multirow[b]{3}{*}{ Sexo } & \multirow[b]{3}{*}{ Media ajustada } & \multirow[b]{3}{*}{ Error típico } & \multirow{2}{*}{\multicolumn{2}{|c|}{ IC95\% }} & \multirow{2}{*}{\multicolumn{3}{|c|}{$\begin{array}{l}\text { Pruebas estadísticas } \\
\text { Modelo Mixto Ajustado }\end{array}$}} \\
\hline & & & & & & & & \\
\hline & & & & Inferior & Superior & $\mathbf{F}$ & $g l$ & $p$ \\
\hline \multirow[t]{2}{*}{$\mathrm{CD}$} & Chica & 5.39 & .07 & 5.25 & 5.54 & \multirow{2}{*}{6.65} & \multirow{2}{*}{408} & \multirow{2}{*}{.010} \\
\hline & Chico & 5.12 & .08 & 4.98 & 5.27 & & & \\
\hline \multirow[t]{2}{*}{ IRR } & Chica & 1.91 & .06 & 1.77 & 2.01 & \multirow{2}{*}{10.75} & \multirow{2}{*}{408} & \multirow{2}{*}{.001} \\
\hline & Chico & 2.17 & .07 & 2.02 & 2.29 & & & \\
\hline \multirow[t]{2}{*}{ DES } & Chica & 1.55 & .06 & 1.43 & 1.66 & \multirow{2}{*}{14.60} & \multirow{2}{*}{408} & \multirow{2}{*}{.000} \\
\hline & Chico & 1.85 & .06 & 1.74 & 1.96 & & & \\
\hline \multirow[t]{2}{*}{ PAC } & Chica & 1.29 & .05 & 1.18 & 1.39 & \multirow{2}{*}{9.57} & \multirow{2}{*}{408} & \multirow{2}{*}{.002} \\
\hline & Chico & 1.51 & .05 & 1.41 & 1.61 & & & \\
\hline \multirow[t]{2}{*}{ BAP } & Chica & 1.23 & .06 & 1.13 & 1.34 & \multirow{2}{*}{27.19} & \multirow{2}{*}{408} & \multirow{2}{*}{.000} \\
\hline & Chico & 1.65 & .06 & 1.53 & 1.75 & & & \\
\hline
\end{tabular}

IC = Intervalo de confianza; $g l=$ grados de libertad; $\mathrm{CD}=$ Competencia docente; IRR = Irresponsabilidad y bajo compromiso; $\mathrm{DES}=$ Desobediencia de las normas; $\mathrm{PAC}=$ Perturbador del ambiente de clase; $\mathrm{BAP}=$ Bajo autocontrol personal. 


\section{Discusión}

El presente estudio planteó los siguientes objetivos: i) Analizar la invarianza factorial por sexo del Cuestionario de Conductas Disruptivas en el aula de Educación Física y de la Escala de Evaluación de la Competencia Docente en Educación Física; ii) Analizar las diferencias según la variable sexo en las conductas disruptivas en el aula de EF y en la evaluación de la competencia docente en EF.

Atendiendo a los resultados obtenidos en el CCDEF, los comportamientos de irresponsabilidad y bajo compromiso obtuvieron los valores más altos y los de perturbar el ambiente de clase, las puntuaciones más bajas. Resultados similares se encontraron en adolescentes españoles y mexicanos (Baños, 2020a; Baños et al., 2018). Sin embargo, perturbar el ambiente de clase se dio con más frecuencia en adolescentes estadounidenses, según se informó en los estudios de Cothran y Kulinna (2007) y de Krech et al. (2010); esta diferencia pudiera ser debida a las características contextuales y culturales propias de cada país. En cuanto a la ETCS-EF, los estudiantes percibieron competentes al profesorado de $\mathrm{EF}$, al igual que otros estudios realizados también con alumnado español (Baños et al., 2019; Granero-Gallegos et al., 2020).

En cuanto al análisis de invarianza factorial en función del sexo, se confirma la invarianza de las escalas, tanto en las cargas factoriales, en los interceptos, como en la varianza del error. Con base a los resultados obtenidos en este estudio, se puede considerar el CCDEF y el ETCSEF como instrumentos válidos para realizar estudios de comparación de medias en función de la variable sexo. Dada la solidez en las características psicométricas conocidas del CCDEF (Granero-Gallegos y Baena-Extremera, 2016; Granero-Gallegos et al., 2020) y del ETCS-EF (Baena-Extremera et al., 2015; Baños, Baena-Extremera y Ortiz-Camacho, 2019), así como la utilización en otras lenguas de las escalas originales (i.e., ETCS, Catano y Harvey, 2011; i.e., PECI, Krech et al., 2010), los datos obtenidos contribuyen a confirmar la validez de constructo. Aunque anteriormente se había realizado análisis de invarianza factorial en función del nivel académico con la escala original de conductas disruptivas (PECI) en estudiantes estadounidenses (Krech et al., 2010), una interesante novedad del presente estudio es que aporta los resultados de invarianza factorial en función del sexo, no habiéndose realizado anteriormente dicho análisis con las escalas de conductas disruptivas y competencia docente.

Los resultados de esta investigación únicamente relacionaron de forma negativa las competencias docentes con conductas de irresponsabilidad y bajo compromiso, sin encontrar relación con los demás comportamientos negativos en el aula, encontrando resultados similares con otros estudios (Granero-Gallegos et al., 2020). Sin embargo, otros trabajos si hallaron relación entre las competencias docentes y las conductas disruptivas (Baños et al., 2019), por lo que parece necesario un mayor análisis sobre los comportamientos negativos en la clase de EF y su relación con las competencias del profesorado de EF.

Por otro lado, se analizaron las diferencias de las variables en función del sexo, encontrando diferencias significativas en todas las dimensiones. En el CCDEF, las chicas adolescentes obtuvieron valores medios más bajos que los chicos, obteniendo resultados similares a los presentados en otros estudios (Baños, 2020b; Beaman, Wheldall y Kemp, 2006; Cothran y Kulinna, 2007; Driessen, 2011; Granero-Gallegos et al., 2020; Kulina et al., 2006). Estos resultados pueden deberse a varios factores. Por un lado, los adolescentes se muestran más competitivos con sus compañeros y desafiantes con el profesorado, buscando captar la atención de las chicas 
(Baños, Barretos-Ruvalcaba, y Baena-Extremera, 2019) y, por otra parte, las chicas muestran conductas más positivas y menos problemáticas (Driessen, 2011), ya que suelen tener comportamientos más introvertidos, mostrándose menos participativas, tímidas y evitando trabajar en grupo (Glock y Kleen, 2017). Por ello, el profesorado de EF afirma encontrar mayores dificultades de manejar los comportamientos de los chicos (Jackson y Smith, 2000; McClowry et al., 2013).

En cuanto al ETCS-EF, las chicas mostraron percibir más competente al profesorado de EF en comparación a los chicos. Resultados similares hallaron otras investigaciones que, aunque las chicas percibieron más competentes a los docentes, no hallaron diferencias significativas en función del sexo (Baños et al., 2019; Granero-Gallegos et al., 2020). Estos resultados pudieran explicarse debido a un mayor apoyo emocional dedicado a las adolescentes por parte del profesorado (Shin y Ryan, 2017), ya que ellos reportan con mayor frecuencia comportamientos de oposición emocional que las chicas (Mullola et al., 2012). De ahí la importancia de que los docentes de EF adquieran gran cantidad de habilidades, no solo en la educación emocional, sino también en el control y manejo de las sesiones (Fortuin, van Geel, y Vedder, 2015; Shin y Ryan, 2014). Sin embargo, son varios los docentes que reportan altos niveles de preocupación sobre cómo manejar efectivamente los problemas de disciplina en el aula (Tsouloupas, Carson, Matthews, Grawitch, y Barber, 2010) ya que se sienten incompetentes ante ciertas situaciones y esto se puede relacionar con el fracaso académico (Jurado-de-los-Santos y Tejeda-Fernández, 2019), con la insatisfacción en la escuela y en la vida (Baños et al., 2019).

En general, a la vista de los resultados de este trabajo, se pueden realizar algunas recomendaciones. Por ejemplo, la creación o fortalecimiento de Aulas de Convivencia en los centros que inviten al alumnado a la reflexión y que los estudiantes sean ayudados y acompañados por otros estudiantes seleccionados para ello. De esta manera, sería interesante fortalecer el Plan de Convivencia de los institutos, así como mejorar la motivación entre los estudiantes, sobre entre los chicos, que son los que presentan peores comportamientos en el aula. Relacionado con esto, también es necesario potenciar la competencia docente entre el profesorado en diferentes aspectos, como la comunicación, conciencia social, organización, y resolución de conflictos en el aula.

A modo de conclusión, los resultados obtenidos del presente estudio identifican que el CCDEF y el ETCS-EF son instrumentos apropiados y válidos para medir, tanto los comportamientos negativos de los estudiantes en el aula de EF, como la competencia docente, independientemente del sexo del alumnado. Además, destacar que los chicos obtuvieron puntuaciones significativamente más altas en conductas disruptivas que las chicas, mientras que ellas percibieron más competente al profesorado de EF que los chicos.

Como fortalezas, destacar la temática investigada, ya que puede contribuir a dar respuesta a una de las principales problemáticas que se encuentran día a día en los centros de educación secundaria. No obstante, a pesar del interés de la temática de este estudio, deben ser tenidas en cuenta algunas limitaciones. La muestra está compuesta por alumnado de secundaria y no se realizó un diseño muestral probabilístico, por lo que los resultados no pueden ser generalizados. El diseño de la investigación no permite profundizar en las causas de los malos comportamientos por lo que sería interesante plantear nuevos diseños en futuros estudios. Por ejemplo, diseños experimentales con programas de intervención focalizados en la responsabilidad personal y social, y en la reducción de las conductas disruptivas. Asimismo, deberían ser consideradas otras variables, y otros diseños mixtos de investigación (i.e., cuantitativos y cualitativos) que permitan 
profundizar más en la raíz de los problemas de comportamiento y en la formación y competencia docente para abordar estos conflictos en el aula.

\section{Referencias}

Baena-Extremera, A., Granero-Gallegos, A., Pérez-Quero, F. J., Bracho-Amador, C., y SánchezFuentes. J. A. (2013). Motivation and motivational climate as predictors of perceived importance of physical education in Spain. South African Journal for Research in Sport, Physical Education and Recreation, 35(2), 1-13.

Baena-Extremera, A., Granero-Gallegos, A., y Martínez-Molina, M. (2015). Validación española de la Escala de Evaluación de la Competencia Docente en Educación Física de secundaria. Cuadernos de Psicología del Deporte, 15(3), 113-122.

Baños, R. (2020a). Clima motivacional y conductas disruptivas en Educación Física en estudiantes españoles y mexicanos de educación secundaria. Journal of Sport and Health Research, in-press.

Baños, R., (2020b). Aburrimientos y comportamientos disruptivos en el aula de Educación Física. Journal of Sport and Health Research, in-press.

Baños, R. Baena-Extremera, A., Ortiz-Camacho, M. M., Zamarripa, J., Beltrán, A., y JuveraPortilla, J. L. (2019). Influencia de las competencias del profesorado de secundaria en los comportamientos disruptivos en el aula. Espiral. Cuadernos del Profesorado, 12(24), 3-10. DOI: $10.25115 /$ ecp.v12i24.21

Baños, R., Barretos-Ruvalcaba, M., y Baena-Extremera, A. (2019). Protocolo de estudio de las variables académicas, psicológicas y de actividad física que influyen en el rendimiento académico de adolescentes mexicanos y españoles. Espiral. Cuadernos del Profesorado, 12(25), 89-99. DOI: 10.25115/ecp.v12i25.2480

Baños, R., Ortiz-Camacho, M. M., Baena-Extremera, A., y Tristán-Rodríguez, J. L. (2017). Satisfacción, motivación y rendimiento académico en estudiantes de Secundaria y Bachillerato. Espiral. Cuadernos del Profesorado, 10(20), 40-50. DOI:10.25115/ ecp.v10i20.1011

Baños, R., Ortiz-Camacho, M. M., Baena-Extremera, A., y Zamarripa, J. (2018). Efecto del género del docente en la importancia de la Educación Física, clima motivacional, comportamientos disruptivos, la intención de práctica futura y rendimiento académico. Retos, 33, 252-257.

Beaman, R., Wheldall, K., y Kemp, C. (2006). Differential teacher attention to boys and girls in the classroom. Educational Review, 58 (3), 339-366. DOI: 10.1080/00131910600748406

Catano, V. M. y Harvey, S. (2011). Student perception of teaching effectiveness: development and validation of the Evaluation of Teaching Competencies Scale (ETCS). Assessment and Evaluation in Higher Education, 36(6), 701-717. doi:10.1080/02602938.2010.484879

Chen, F. F. (2007). Sensitivity of goodness of fit indexes to lack of measurement invariance. StructuralEquationModeling, 14(3),464-504.https://doi.org/10.1080/10705510701301834

Colinchón, M. E. (2020). Inteligencia emocional y habilidades sociales en la conducta disruptiva de estudiantes del nivel secundario. Espiral. Cuadernos del Profesorado, 13(26). DOI: 10.25115/ecp.v13i26

Cothran D. J., Kulinna P. H., y Garrahy, D. (2009). Attributions for and consequences of student misbehavior. Physical Education and Sport Pedagogy, 14, 155-167. DOI: 10.1080/17408980701712148 
Cothran,D.J.yKulinna,P.H.(2007).Students'reports ofmisbehaviorinphysicaleducation.Research Quarterly for Exercise and Sport, 78(3), 216-224. DOI: 10.1080/02701367.2007.10599419

Declaración de Helsinki (2008). World Medical Association. Available at http://www.wma.net/ es/30publications/10policies/b3/.

Driessen, G. (2011). Gender differences in education: Is there really a "boys 'problem"? Paper presented at the Annual Meeting. ECER: Berlin, Germany. Accessed from: https://www. researchgate.net/publication/236004056_Gender_differences_in_education_Is_there really_a_boys'_problem

Fortuin, J., van Geel, M., y Vedder, P. (2015). Peer influences on internalizing and externalizing problems among adolescents: A longitudinal social network analysis. Journal of Youth and Adolescence, 44, 887- 897. DOI: 10.1007/s10964-014-0168-x

Fredericks, J. A., Blumenfeld, P. C., y Paris, A. H. (2004). School engagement: Potential of the concept, state of the evidence. Review of Educational Research, 74, 59-109. DOI: $10.3102 / 00346543074001059$

Gelman, A. y Hill, J. (2006). Data Analysis Using Regression and Multilevel/Hierarchical Models. Cambridge: University Press. doi: 10.1017/CBO9780511790942

Glock, S. y Kleen, H. (2017). Gender and student misbehavior: Evidence from implicit and explicit measures. Teaching and Teacher Education, 67, 93-103. DOI: 10.1016/j.tate.2017.05.015

Goyette, R., Dore, R., y Dion, E. (2000). Pupils' misbehaviors and the reactions and causal attributions of physical education student teachers: A sequential analysis. Journal of Teaching in Physical Education, 20, 3-14. DOI: 10.1123/jtpe.20.1.3

Granero-Gallegos, A. y Baena-Extremera, A. (2016). Validación española de la version corta del Physical Education Classroom Instrument para la medición de conductas disruptivas en alumnado de secundaria. Cuadernos de Psicología del Deporte, 16(2) 89-98.

Granero-Gallegos, A., Baños, R., Baena-Extremera, A., y Martínez-Molina, M. (2020). Analysis of misbehaviours and satisfaction with school in secondary education according to student gender and teaching competence. Frontiers in Psychology, 11(63), 1-9. DOI: 10.3389/fpsyg.2020.00063

Granero-Gallegos, A., Gómez-López, M., Abraldes, J. A. y Rodríguez-Suárez, N. (2011). Motivos de práctica en el ámbito de la actividad física no competitiva. Espiral. Cuadernos del Profesorado, 4(7), 15-22. DOI: 10.25115/ecp.v4i7.915

Granero-Gallegos, A., Gómez-López, M., Baena-Extremera, A., y Martínez-Molina, M. (2019a). Interaction Effects ofDisruptive Behaviour and Motivation Profiles with TeacherCompetence and School Satisfaction in Secondary School Physical Education. International Journal of Environmental Research and Public Health, 17(1), 114. DOI: 10.3390/ijerph17010114

Granero-Gallegos, A., Ruiz-Montero, P. J., Baena-Extremera, A., y Martínez-Molina, M. (2019b). Effects of Motivation, Basic Psychological Needs, and Teaching Competence on Disruptive Behaviours in Secondary School Physical Education Students. International Journal of Environmental Research and Public Health, 16(23), 4828. DOI: 10.3390/ijerph16234828

Hox, J. J. (1998). Multilevel modeling: When and why. In I. Balderjahn, R. Mathar, y M. Schader (Eds.), Classification, data analysis and data highways (pp. 147-155). Berlin: Springer Berlin Heidelberg. Jackson, C. y Smith, I. D. (2000). Polesapart? An exploration of single-sex educational environments in Australia and England. Educational Studies, 26(4),409-422. DOI: $10.1080 / 03055690020003610$

(C) Psy, Soc, \& Educ, 2020, Vol. 12(2) 
Jurado-de-los-Santos, P., y Tejada-Fernández, J. (2019). Disrupción y fracaso escolar. Un estudio en el contexto de la Educación Secundaria Obligatoria en Cataluña. Estudios sobre Educación, 36, 135-155. DOI: 10.15581/004.36.135-155

Krech, P. R., Kulinna, P. H., y Cothran, D. (2010). Development of a short-form version of the PhysicalEducationClassroomInstrument:measuringsecondarypupils'disruptivebehaviours. Physical Education Sport Pedagogy, 15(3), 209-225. doi: 10.1080/17408980903150121

Kulinna P. H., Cothran D., y Regualos R. (2006). Teachers' reports of student misbehavior in physical education. Research Quarterly for Exercise and Sport, 77, 32-40.

McClowry, S. G., Rodriguez, E. T., Tamis-LeMonda, C. S., Spellmann, M. E., Carlson, A., y Snow, D. L. (2013). Teacher/student interactions and classroom behavior: The role of student temperament and gender. Journal of Research in Childhood Education, 27(3), 283301. DOI: $10.1080 / 02568543.2013 .796330$

Medina, J. A. y Reverte, M. J. (2019). Incidencia de la práctica de actividad física y deportiva como reguladora de la violencia escolar. Retos, 35, 54-60.

Milfont, T. L. y Fisher, R. (2010). Testing measurement invariance across groups: Applications in cross. International Journal of Psychological Research, 3(1), 111-121. DOI: 10.21500/20112084.857

Müller, C. M., Hofmann, V., Begert, T., y Cillessen, A. H. (2018). Peer influence on disruptive classroom behavior depends on teachers' instructional practice. Journal of Applied Developmental Psychology, 56, 99-108. DOI: 10.1016/j.appdev.2018.04.001

Mullola, S., Ravaja, N., Lipsanen, J., Alatupa, S., Hintsanen, M., Jokela, M., y KeltikangasJärvinen, L. (2012). Gender differences in teachers' perceptions of students' temperament, educational competence, and teachability. British Journal of Educational Psychology, 82(2), 185-206. DOI: 10.1111/j.2044-8279.2010.02017.x

Pardo, A., Ruiz, M., y San Martín, R. (2007). Cómo ajustar e interpretar modelos multinivel con SPSS. Psicothema, 19(2), 308-321.

Rasmussen, J. F., Scrabis-Fletcher, K., y Silverman, S. (2014). Relationships among tasks, time, and student practice in elementary physical education. Physical Educator, 71(1), 114-131.

Shin, H. y Ryan, A. M. (2014). Friendship networks and achievement goals: An examination of selection and influence processes and variations by gender. Journal of Youth and Adolescence, 43, 1453-1464. DOI: 10.1007/s10964-014-0132-9

Shin, H. y Ryan, A. M. (2017). Friend influence on early adolescent disruptive behavior in the classroom: Teacher emotional support matters. Developmental Psychology, 53(1), 114-126. DOI: $10.1037 / \operatorname{dev} 0000250$

Tsouloupas, C. N., Carson, R. L., Matthews, R., Grawitch, M. J., y Barber, L. K. (2010). Exploring the association between teachers' perceived student misbehaviour and emotional exhaustion: the importance of teacher efficacy beliefs and emotion regulation. Educational Psychology, 30(2), 173-189. DOI: 10.1080/01443410903494460

Weiss, M. R., Smith, A. L., y Stuntz, C. P. (2008). Moral development in sport and physical activity. In T. S. Horn (Ed.), Advances in Sport Psychology (pp. 187-210). Champaign, IL, US: Human Kinetics. 